INFLAMMATORY BOWEL DISEASE

\title{
Is inflammatory bowel disease an independent and disease specific risk factor for thromboembolism?
}

\author{
W Miehsler, W Reinisch, E Valic, W Osterode, W Tillinger, T Feichtenschlager, J Grisar, K Machold, \\ S Scholz, H Vogelsang, G Novacek
}

Background: Patients with inflammatory bowel disease (IBD) are thought to be at increased risk of venous thromboembolism (TE). However, the extent of this risk is not known. Furthermore, it is not known if this risk is specific for IBD or if it is shared by other chronic inflammatory diseases or other chronic bowel diseases. Aims: To compare the risk of TE in patients with IBD, rheumatoid arthritis, and coeliac disease with matched control subjects.

See end of article for authors' affiliations Patients and methods: Study subjects answered a questionnaire assessing the history of TE, any cases of which had to be confirmed radiologically. A total of 618 patients with IBD, 243 with rheumatoid arthritis, 207 with coeliac disease, and 707 control subjects were consecutively included. All three patient groups were compared with control subjects matched to the respective group by age and sex.

Correspondence to: Professor G Novacek, University of Vienna, Department of Internal Medicine IV, Division of Gastroenterology and Hepatology, Waehringer Guertel 18-20, 1090

Vienna, Austria;

Gottfried.Novacek@ akh-wien.ac.at

Accepted for publication 11 September 2003

Results: Thirty eight IBD patients (6.2\%) had suffered TE. This was significantly higher compared with the matched control population with only 10 cases reported (1.6\%) $(p<0.001$; odds ratio (OR) 3.6 (95\% confidence interval (CI) 1.7-7.8)). Five patients with rheumatoid arthritis (2.1\%) had suffered TE compared with six subjects $(2.5 \%)$ in the control population matched to patients with rheumatoid arthritis (NS; OR 0.7 (95\% Cl 0.2-2.9)). TE had occurred in two patients with coeliac disease (1\%) compared with four subjects $(1.9 \%)$ in the control population matched to the coeliac disease group (NS; OR $0.4(95 \% \mathrm{Cl} 0.1-2.5)$ ). In $60 \%$ of TE cases in the IBD group, at least one IBD specific factor (active disease, stenosis, fistula, abscess) was present at the time TE occurred.

Conclusions: IBD is a risk factor for TE. It seems that TE is a specific feature of IBD as neither rheumatoid arthritis, another chronic inflammatory disease, nor coeliac disease, another chronic bowel disease, had an increased risk of TE.

$\mathrm{T}$ hromboembolism (TE) is a serious and potentially life threatening event. For many years, patients with inflammatory bowel disease (IBD) have been thought to be at increased risk of TE. ${ }^{1-6}$ Studies using different methodological approaches have revealed conflicting data on the prevalence of TE in IBD, varying between $1.2 \%$ and $6.7 \%$ in clinical studies and rising to $39 \%$ in postmortem studies. However, methodological shortcomings in these studies, such as lack of control group or diagnostic weakness regarding TE and IBD, have thus far made it almost impossible to determine whether or not IBD carries a higher risk of TE.

Furthermore, it is not known whether this suspected increased risk of TE is a specific feature of IBD or if it is also associated with chronic inflammatory diseases or chronic bowel diseases other than IBD. Rheumatoid arthritis is one example of a chronic inflammatory disease, and thromboembolic events have been described in rheumatoid arthritis patients, especially in association with antiphospholipid antibodies. ${ }^{7}$ Additionally, TE has been reported in rheumatoid arthritis as a postoperative complication after arthroplasty. ${ }^{8}$ Coeliac disease is a chronic bowel disease in which TE has occasionally been reported. Some cases were associated with hyperhomocysteinaemia, possibly caused by impaired absorption of regulating vitamins. ${ }^{910}$ However, for both diseases, rheumatoid arthritis and coeliac disease, the prevalence of $\mathrm{TE}$ and the risk of TE compared with control subjects is not known.

This study was designed to investigate if patients with IBD are at increased risk of TE compared with control subjects and, if they are, whether or not this increased risk is specific for IBD, or if it is also found in patients with rheumatoid arthritis or coeliac disease.

\section{METHODS}

A total of 618 patients with IBD, 243 with rheumatoid arthritis, 207 with coeliac disease, and 707 control subjects were consecutively included. All three patient groups were compared with control subjects matched to the respective group by age and sex in a $1: 1$ fashion. Study subjects answered a questionnaire assessing the prevalence of TE. Study subjects were eligible if they were at least 18 years of age and had no history of malignant disease. Any event of TE had to be confirmed by imaging procedures. The study was approved by the ethics committee of the University of Vienna.

\section{Patients with inflammatory bowel disease}

Patients with IBD were recruited consecutively from three outpatient clinics of the Divisions of Gastroenterology and Hepatology: (1) Department of Internal Medicine IV, University of Vienna; (2) Department of Internal Medicine 4, Krankenhaus Rudolfstiftung, Vienna (general hospital); and (3) Department of Internal Medicine 1, Krankenhaus Lainz, Vienna (general hospital). The diagnosis of IBD was based on clinical, endoscopic, histological, and radiological

Abbreviations: IBD, inflammatory bowel disease; $\mathrm{TE}$, thromboembolism; CD, Crohn's disease; UC, ulcerative colitis; IC, indeterminate colitis; OR, odds ratio; TNF- $\alpha$, tumour necrosis factor $\alpha$; $\mathrm{BMI}$, body mass index 


\begin{tabular}{|c|c|c|c|c|}
\hline & $\begin{array}{l}C D \\
(n=485)\end{array}$ & $\begin{array}{l}U C \\
(n=125)\end{array}$ & $\begin{array}{l}\text { IC } \\
(n=8)\end{array}$ & $\begin{array}{l}\text { Total } \\
(n=618)\end{array}$ \\
\hline Age $(y)^{*}$ & $38.1(12.4)$ & $43.4(16.0)$ & $33.0(13.5)$ & $39.0(13.0)$ \\
\hline \multicolumn{5}{|l|}{ Sex } \\
\hline Male (n) & 213 & 62 & 5 & 280 \\
\hline Female (n) & 272 & 63 & 3 & 338 \\
\hline Duration of disease $(y)^{*}$ & $9.3(7.4)$ & $9.6(8.0)$ & $5.3(3.9)$ & $9.3(7.5)$ \\
\hline \multicolumn{5}{|l|}{ Disease distribution (n) } \\
\hline Small bowel & 75 & & & \\
\hline Large bowel & 73 & & & \\
\hline Small+large bowel & 337 & & & \\
\hline Proctosigmoid & & 30 & 2 & \\
\hline Left sided & & 42 & 3 & \\
\hline Pancolitis & & 53 & 3 & \\
\hline \multicolumn{5}{|l|}{ Resections (n) } \\
\hline None & 211 & 118 & 8 & 337 \\
\hline Small bowel & 74 & & & \\
\hline Large bowel & 25 & & & \\
\hline Small+large bowel & 175 & & & \\
\hline Proctosigmoid & & 6 & & \\
\hline Colectomy & & 1 & & \\
\hline
\end{tabular}

criteria according to Lennard-Jones and Shivananda. ${ }^{11}$ IBD was classified as Crohn's disease (CD), ulcerative colitis (UC), and indeterminate colitis (IC). Type of IBD, duration of disease, disease distribution, and type of bowel resections were determined by review of hospital records in all patients (table 1).

\section{Controls}

Controls were recruited consecutively from the outpatient clinic of the Division of Occupational Medicine, Department of Internal Medicine IV, University of Vienna. Subjects attend this department for their regular preventive medical check up for exposure to solvents (xylene, toluene, etc), heavy metals (lead, mercury, cadmium, etc), radiation, or noise.

\section{Patients with rheumatoid arthritis}

Patients with rheumatoid arthritis were recruited consecutively from the outpatient clinic of the Division of Rheumatology, Department for Internal Medicine III, University of Vienna. The diagnosis of rheumatoid arthritis was based on the revised criteria for a diagnosis of rheumatoid arthritis by the American Rheumatism Association. ${ }^{12}$

\section{Patients with coeliac disease}

Patients with coeliac disease were recruited consecutively from the outpatient clinic specialising in coeliac disease at the Division of Gastroenterology and Hepatology, Department for Internal Medicine IV, University of Vienna. The diagnosis of coeliac disease was based on typical morphology of duodenal mucosal biopsy specimens taken during oesophago-gastroduodenoscopy, according to the revised criteria of WalkerSmith and colleagues. ${ }^{13}$

\section{Questionnaire}

To assess the prevalence of TE, all study subjects were required to complete a questionnaire distributed by the respective physician during the patient's visit to the respective outpatient clinic. TE was explained to the study subjects in a "common sense" manner, and they were asked if they had ever experienced it. After assessment of demographic data, including calculation of body mass index (BMI), study subjects answered the questionnaire, which was structured into three sections, each covering several items, most to be answered as multiple choice questions.
The first section asked "Have you ever had a thromboembolic event such as: deep venous thrombosis, pulmonary embolism, thrombosis of the lower caval vein, thrombosis of the arm vein, thrombosis of the mesenteric vein, or a venous thrombosis of any other location". As a back up question, patients were additionally asked if they had ever received anticoagulant therapy and for which indication and duration this was taken. The second section assessed the history of known risk factors for TE. Patients were asked in detail for the number and type of operations, number and type of injuries and period of immobility (including cast immobility), pregnancies, time of oral contraceptive use or hormone substitution, or if a TE had occurred within four weeks of a long distance journey ( $\geqslant 4$ hours by plane, bus, or car). ${ }^{14}$ Each item was followed by the question if TE had occurred in relation to the respective item (time frame of six weeks for postoperative thrombosis). The third section asked about the presence of any other diseases and smoking habits.

\section{Diagnosis of thromboembolism}

Only venous thromboembolic events that had been diagnosed by an imaging procedure were counted. Diagnosis of venous thrombosis of the leg or arm was accepted if established by phlebography or duplex ultrasonography. Diagnosis of pulmonary embolism was accepted if established by helical computed tomography of the chest or pulmonary scintillation scanning. ${ }^{15}$ Where pulmonary embolism had occurred with concomitant deep venous thrombosis, the thromboembolic event was counted as pulmonary embolism only. Thrombosis of the mesenteric vein was diagnosed by contrast enhanced computed tomography of the abdomen.

Furthermore, we wished to evaluate a potential association between occurrence of TE and IBD specific factors, such as disease activity, and presence of stenosis, fistula, or abscess. Therefore, if a thromboembolic event in an IBD patient was confirmed, the complete hospital records of the IBD care centre and of the hospital where the TE was diagnosed were reviewed in order to determine the status of the underlying IBD at the time TE occurred. Disease activity at the time of TE was reassessed using the Harvey-Bradshaw index (active $\geqslant 4$ points) ${ }^{16}$ or the Truelove-Witts criteria (active if classified as "moderate" or "severe"), ${ }^{17}$ respectively. Presence of fistula at the time of TE was assumed if so stated in the hospital file. Presence of bowel stenosis at the time of TE was assumed if assessed by enteroclysis, large bowel enema, or colonoscopy 
within six months prior to TE. Presence of abscesses at the time of TE were assumed if documented incision or drainage of an abscess had been performed within two weeks before or after TE. Presence of a central venous catheter was assumed if definitely stated in the hospital files.

\section{Statistical analysis}

Statistical analysis and consulting for study design was done by the Department of Medical Statistics, University of Vienna. The SAS software package (SAS, North Carolina, USA) was used for statistical calculations. Differences between groups were analysed using the Student's $t$ test or $\chi^{2}$ test as appropriate. The relative risk of having TE was expressed by odds ratios (OR) with $95 \%$ confidence intervals (95\% CI). Survival without having TE was compared between IBD and control subjects using Kaplan-Meier survival analysis with the log rank test to test the equality of the survival curves. Based on the literature ${ }^{1-6}$ and our own experience, we expected that the prevalence of TE in our outpatients with IBD would be at least 3\%. The prevalence of $\mathrm{TE}$ in an Austrian background population was estimated based on the incidence data by Silverstein et al taking into account the expected age distribution, and was assumed to be $1 \%{ }^{18}$ This estimation was recently confirmed by prevalence data in a central European study. ${ }^{19}$ Type I error was defined as a one tailed $\alpha$ level of 0.05 . Type II error was defined as a $\beta$ level of 0.2 at a statistical power of 0.8 . We calculated that we needed at least 605 patients in the IBD group and 605 controls to detect a significant difference. ${ }^{20}$ The study design included 1:1 matching with regard to sex and age ( \pm 3 years), primarily for IBD patients. For the rheumatoid arthritis and coeliac disease groups, matching was done retrospectively: each patient with rheumatoid arthritis or coeliac disease was matched to a control subject of the same sex closest to age. To evaluate significant risk factors, logistic regressions were performed and ORs were adjusted for these risk factors.

\section{RESULTS}

Prevalence of thromboembolism (see tables 2-4)

IBD versus control group (see table 2)

Thirty eight IBD patients $(6.15 \%)$ had a history of radiologically proven TE which was significantly higher compared with the control group matched to the IBD patients in which only 10 subjects had suffered a TE (1.62\%) (OR 3.6 (95\% CI 1.7-7.8), $\mathrm{p}<0.001$; odds ratio adjusted for the risk factors operation, injuries, oral contraceptive use, pregnancy, BMI, and smoking in the logistic regression model). Kaplan-Meier analysis showed that with increasing age, a significantly lower proportion of IBD patients remained free of TE compared with control subjects $(p<0.001)$, with a substantial decline in this proportion after the age of 45 years (fig 1). Nineteen of 38 IBD patients with TE $(50 \%)$ and four of 10 control subjects with TE were women (40\%; NS). The median duration of IBD at the time of the first TE was 5 years (range $0-28)$. No significant difference in the prevalence of TE was found between CD $(27 / 485,5.6 \%)$, UC $(11 / 125,8.8 \%)$, and IC $(0 / 8)$.

\section{Rheumatoid arthritis versus control group (see table 3)}

Five patients $(2.06 \%)$ in the rheumatoid arthritis group had a history of proven TE, similar to the control group matched to the rheumatoid arthritis group in which six subjects $(2.5 \%)$ had a history of TE (NS; OR 0.7 (95\% CI 0.2-2.9); odds ratio adjusted for the risk factors operation, injuries, oral contraceptive use, and BMI in the logistic regression model). Median duration of rheumatoid arthritis at the time of the first TE was 3 (range 0-28) years. In the rheumatoid arthritis group, all TE occurred in women whereas in the control group TE occurred in three women and three men.

\section{Coeliac disease versus control group (see table 4)}

Two patients with coeliac disease $(1.0 \%)$ had a history of proven TE, similar to the control group matched to the coeliac disease group in which four subjects $(1.9 \%)$ had a history of TE (NS; OR 0.4 (95\% CI 0.1-2.5); odds ratio adjusted for the risk factors pregnancy, BMI, and smoking in the logistic regression model). In the coeliac disease group, both TE cases occurred in women whereas in the control group TE occurred in two women and two men.

\section{Types of TE (table 5)}

The 38 IBD patients had experienced 47 thromboembolic events. Thirty IBD patients had suffered one TE, seven two TEs, and one three TEs. The two dominant types of TE were deep venous thrombosis and pulmonary embolism, responsible for $79 \%$ of thromboembolic events in the IBD group. In the control group, nine subjects had experienced one TE and one subject had experienced three TEs. All patients with rheumatoid arthritis and coeliac disease who had a history of TE had one TE. All TEs in the control group as well as in the rheumatoid arthritis and coeliac disease groups were deep venous thromboses or pulmonary embolisms.

Table 2 Characteristics of patients with inflammatory bowel disease (IBD) compared with controls matched to the IBD patients for age and sex, outlining the prevalence of venous thromboembolism (TE) and clinical risk factors for venous TE

\begin{tabular}{|c|c|c|c|}
\hline & $\begin{array}{l}\text { IBD } \\
(n=618)\end{array}$ & $\begin{array}{l}\text { Control subjects } \\
(n=618)\end{array}$ & $\mathrm{p}$ Value \\
\hline Age $(y)^{\star}$ & $39.1(13.4)$ & $39.1(13.3)$ & Matched \\
\hline \multicolumn{4}{|l|}{ Sex } \\
\hline Male/female (n) & $280 / 338$ & $280 / 338$ & Matched \\
\hline \multicolumn{4}{|l|}{ TE } \\
\hline No (\%) & $38(6.15)$ & $10(1.62)$ & $<0.001$ \\
\hline Male/female (n) & $19 / 19$ & $6 / 4$ & \\
\hline No of operations* & $2.3(1.8)$ & $1.4(1.3)$ & $<0.001$ \\
\hline No of injuries* & $0.6(0.7)$ & $0.6(0.8)$ & \\
\hline Oral contraceptive use $\dagger(n)$ & 257 & 222 & 0.002 \\
\hline Duration of oral contraceptive use $(y)^{*}$ & $10.2(7.7)$ & $7.0(5.4)$ & $<0.001$ \\
\hline Pregnancies* & $1.4(1.5)$ & $1.2(1.4)$ & 0.037 \\
\hline $\mathrm{BMl}^{*}$ & $23.2(4.2)$ & $24.1(4.1)$ & $<0.001$ \\
\hline Smoker (n) & 392 & 317 & $<0.001$ \\
\hline
\end{tabular}

*Values are mean (SD).

tIncludes hormone substitution.

$\mathrm{BMI}$, body mass index. 
Table 3 Characteristics of patients with rheumatoid arthritis compared with controls matched to the rheumatoid arthritis patients for age and sex, outlining the prevalence of venous thromboembolism (TE) and clinical risk factors for venous TE

\begin{tabular}{|c|c|c|c|}
\hline & $\begin{array}{l}\text { Rheumatoid arthritis } \\
\text { ( } n=243 \text { ) }\end{array}$ & $\begin{array}{l}\text { Control subjects } \\
(n=243)\end{array}$ & p Value \\
\hline Age $(y)^{*}$ & $54.6(14.2)$ & $50.6(10.9)$ & NS \\
\hline Sex & & & \\
\hline Male/female (n) & $48 / 195$ & $48 / 195$ & Matched \\
\hline No $(\%)$ & $5(2.06)$ & $6(2.47)$ & NS \\
\hline Male/female (n) & $0 / 5$ & $3 / 3$ & \\
\hline No of operations* ${ }^{*}$ & $2.5(2.1)$ & $1.8(1.4)$ & $<0.0001$ \\
\hline No of injuries* & $0.5(0.7)$ & $0.6(0.9)$ & 0.0095 \\
\hline Oral contraceptive use $†$ (n) & 92 & 114 & 0.040 \\
\hline Duration of oral contraceptive use $(y)^{*}$ & $10.0(7.7)$ & $9.1(7.0)$ & NS \\
\hline No of pregnancies* & $1.8(1.5)$ & $1.8(1.5)$ & NS \\
\hline $\mathrm{BMI}^{*}$ & $25.2(4.2)$ & $24.5(4.0)$ & NS \\
\hline Smoker (n) & 114 & 110 & NS \\
\hline
\end{tabular}

*Values are mean (SD).

tIncludes hormone substitution.

BMI, body mass index.

Table 4 Characteristics of patients with coeliac disease compared with controls matched to the coeliac disease patients for age and sex, outlining the prevalence of venous thromboembolism (TE) and clinical risk factors for venous TE

\begin{tabular}{|c|c|c|c|}
\hline & $\begin{array}{l}\text { Coeliac disease } \\
(n=207)\end{array}$ & $\begin{array}{l}\text { Control subjects } \\
(n=207)\end{array}$ & $\mathrm{p}$ Value \\
\hline Age $(y)^{*}$ & $42.4(15.4)$ & $42.3(15.1)$ & NS \\
\hline \multicolumn{4}{|l|}{ Sex } \\
\hline Male/female (n) & $49 / 158$ & $49 / 158$ & Matched \\
\hline \multicolumn{4}{|l|}{ TE } \\
\hline No (\%) & $2(0.97)$ & $4(1.93)$ & NS \\
\hline Male/female (n) & $0 / 2$ & $2 / 2$ & \\
\hline No of operations* & $1.4(1.4)$ & $1.5(1.3)$ & NS \\
\hline No of injuries* & $0.7(0.8)$ & $0.7(0.8)$ & NS \\
\hline Oral contraceptive use $(n)$ & 112 & 95 & NS \\
\hline Duration of oral contraceptive use $(y)^{*}$ & $8.0(5.8)$ & $7.0(5.6)$ & NS \\
\hline No of pregnancies* & $1.6(1.5)$ & $1.2(1.4)$ & 0.041 \\
\hline BMI & $22.2(3.1)$ & $23.0(3.4)$ & 0.01 \\
\hline Smoker (n) & 87 & 92 & NS \\
\hline
\end{tabular}

*Values are mean (SD).

tIncludes hormone substitution.

BMI, body mass index.

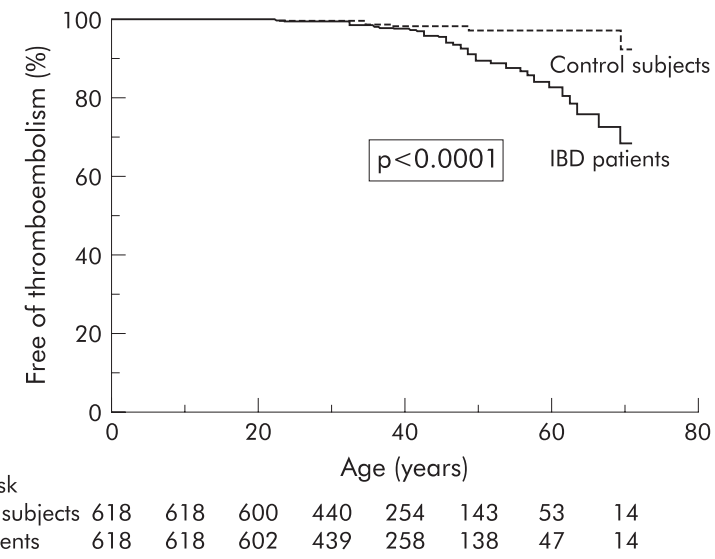

Figure 1 Kaplan-Meier curves for patients with inflammatory bowel disease (IBD) and control subjects, for being free of thromboembolism (TE). With increasing age, a significantly lower proportion of IBD patients remain free of TE compared with control subjects $(p<0.0001$; log rank test), with a substantial decline in this proportion after the age of 45 years.
TE in IBD: risk factors and disease status (table 5)

At least one of the known clinical risk factors for TE (long distance journey, postoperative, injury/immobility, pregnancy/delivery, oral contraceptives/hormone substitution) was present in 13 of 47 TEs in the IBD group (28\%) and in seven of the 12 TEs in control subjects matched to the IBD group $(58 \%)$ at the time TE occurred (see table 5 ). In these 13 IBD cases, one clinical risk factor was present in nine thromboembolic events and two or more clinical risk factors were present in four thromboembolic events. Additionally, at least one IBD specific factor such as active disease, fistula, stenosis, or abscess was present in 28 of 47 thromboembolic events in the IBD group (60\%) at the time TE occurred. In those 28 cases, one IBD specific factor was present in 24 thromboembolic events and two or more IBD specific factors were present in four thromboembolic events. An overlap between the presence of clinical risk factors for TE and IBD specific factors at the time TE occurred was seen in seven thromboembolic events. IBD specific therapy at the time of TE is outlined in table 5 .

\section{DISCUSSION}

We found that patients with IBD have a 3.6-fold higher risk of TE compared with controls matched for age and sex. This 
Table 5 Specifications of thromboembolic events

\begin{tabular}{|c|c|c|c|c|}
\hline & $\begin{array}{l}\text { IBD } \\
(n=618)\end{array}$ & $\begin{array}{l}\text { Controls* } \\
(n=618)\end{array}$ & $\begin{array}{l}\text { Rheumatoid } \\
\text { arthritis } \\
(n=243)\end{array}$ & $\begin{array}{l}\text { Coeliac disease } \\
(n=207)\end{array}$ \\
\hline Patients with TE (n) & $38 \dagger$ & $10 \dagger$ & 5 & 2 \\
\hline One TE & 30 & 9 & 5 & 2 \\
\hline Two TE & 7 & - & - & - \\
\hline Three TE & 1 & 1 & - & - \\
\hline Total number of TEs & 47 & 12 & 5 & 2 \\
\hline Age at first TE (median (range)) & 47 (18-84) & 35 (22-69) & $56(33-64)$ & 34 and 53 \\
\hline \multicolumn{5}{|l|}{ Site of TE $(n)$} \\
\hline Deep vein & 23 & 6 & 5 & 2 \\
\hline $\mathrm{PE}$ & 14 & 6 & - & - \\
\hline Subclavian vein & 4 & - & - & - \\
\hline Arm vein & 3 & - & - & - \\
\hline Otherf & 3 & - & - & - \\
\hline \multicolumn{5}{|l|}{ Clinical risk factors (n) } \\
\hline Flight§ & 3 & 1 & 1 & - \\
\hline Postoperative & 5 & 1 & 1 & - \\
\hline Injury & 2 & 1 & - & 1 \\
\hline Pregnancy & - & 2 & 1 & - \\
\hline OC & 5 & 2 & 1 & - \\
\hline CVC & 5 & - & - & - \\
\hline \multicolumn{5}{|l|}{ IBD specific factors ( $n$ ) } \\
\hline Clinical activity & 18 & & & \\
\hline Fistula & 5 & & & \\
\hline Abscess & 1 & & & \\
\hline Stenosis & 9 & & & \\
\hline \multicolumn{5}{|l|}{ IBD specific therapy $(n)$} \\
\hline 5-ASA & 17 & & & \\
\hline Steroids & 13 & & & \\
\hline AZA & 5 & & & \\
\hline \multicolumn{5}{|c|}{$\begin{array}{l}{ }^{*} \text { Control group matched to the IBD group by age ( } \pm 3 \text { years) and sex. } \\
\dagger \chi^{2} \text { test, } \mathrm{p}<0.001 ; \mathrm{OR}=3.6(95 \% \mathrm{Cl} 1.7-7.8) \text { adjusted for the risk factors operation, injuries, oral contraceptive } \\
\text { use, pregnancy, } \mathrm{BMI} \text {, and smoking in the logistic regression model. } \\
\text { ¥Cavernous sinus thrombosis, mesenteric vein thrombosis, or superior caval vein thrombosis. } \\
\S \text { Travelling by plane, bus or car, } \geqslant 4 \text { hours. } \\
\text { IBD, inflammatory bowel disease; TE, thromboembolism; PE, pulmonary embolism; OC, oral contraceptive use or } \\
\text { hormone substitution; CVC, central venous catheter; } 5 \text {-ASA, 5-aminosalicylic acid; AZA, azathioprine. }\end{array}$} \\
\hline
\end{tabular}

represents a relevant extraintestinal complication of IBD, including life threatening pulmonary embolism.

This increased risk of TE in IBD has long been proposed, with wide variation in the reported prevalences of between $1.2 \%$ and $6.1 \%$ in database queries and chart reviews and even reaching $39 \%$ in necropsy studies. ${ }^{1-6}$ There are limitations concerning the weight of these studies in view of their methodological shortcomings-mainly the lack of representative matched control groups-making their interpretation difficult. This shortcoming, the lack of a control group, was recently overcome in a database query that yielded a relative risk of three for TE in IBD, similar to our results. ${ }^{5}$ However, in this study, patients were not subject to stringent diagnostic criteria. Subjects were considered IBD patients if the diagnosis "IBD" was noted several times in a "physician claim". In fact, it is possible that diagnostic misclassification of IBD could have influenced the reliability of an association between IBD and TE which has in analogy already been shown for genetic linkage studies. ${ }^{21}$ To avoid distortion of the data by diagnostic misclassification, we only enrolled patients in the care of IBD centres as these patients reliably fulfilled stringent diagnostic criteria for IBD..$^{22}$

As back up questions, every item on the clinical risk factors for TE was followed by the repeat question of whether TE had occurred in relation to the respective item. Furthermore, patients were asked if they had ever received anticoagulant therapy and for which indication and duration this was taken. Thus the design of our questionnaire increased the probability that all thromboembolic events that had occurred in our outpatient population were recorded.

The second important result of our study is the fact that the prevalence of TE was not increased in rheumatoid arthritis and coeliac disease, indicating that TE is a specific feature of
IBD. On the one hand, one would expect an increased prevalence of TE in rheumatoid arthritis due to arthroplasty, a common procedure in patients with rheumatoid arthritis and an association with antiphospholipid antibody syndrome. However, in the present study there was no increased prevalence of TE in rheumatoid arthritis. To our knowledge only one retrospective study has mentioned the prevalence of TE in rheumatoid arthritis although it has to be interpreted cautiously as the data were generated only from chart review and there was no control group. ${ }^{24}$ However, the reported prevalence of TE in rheumatoid arthritis was 3.8\%, being higher than in our data, which can be explained by the fact that in this study TE was also accepted on the basis of a clinical diagnosis alone. The prevalence of TE in coeliac disease was $1 \%$, the same as in the control group. Given the fact that coeliac disease occurs independently of socioeconomic status, the coeliac disease group may be seen as an ideal representative sample of the background population, underscoring the validity of our control group. However, we have provided the first data on the prevalence of TE in coeliac disease. However, TE has also been described as the initial symptom in coeliac disease which has been attributed to acquired hyperhomocysteinaemia as a consequence of folic acid and vitamin B12 deficiency. ${ }^{9}{ }^{10}$ Hyperhomocysteinaemia has been recognised as an independent risk factor for arterial and venous TE which may occur secondary to folate and vitamin B12 deficiencies due to malabsorbtion as, for example, in the case of coeliac disease. ${ }^{25}$

Since the risk of TE is substantially higher in patients with IBD compared with rheumatoid arthritis and coeliac disease, the question then is why. Was it an ascertainment bias in favour of the IBD group as one could argue that IBD patients were more likely to have been subjected to imaging 
procedures required to make the diagnosis? For two reasons it is unlikely that a significant ascertainment bias occurred. Firstly, comparable intensity of medical care was provided in all three patient groups and, secondly, a considerable number of TEs in all groups were diagnosed and treated in primary and secondary care institutions in a standard fashion, irrespective of whether or not subjects had IBD. The second question in view of this increased risk is the certainly as to whether or not there is a simple explanation for this result based on the higher prevalence of one of the known clinical risk factors for TE in IBD. On average, patients with IBD have had a higher number of operations, longer duration of oral contraceptive use, more pregnancies, higher proportion of smokers, ${ }^{26}$ and a lower BMI. However, it appears that these factors are not of major relevance for the development of TE in IBD as the relative risk for TE in IBD still remained high after adjustment for these confounding variables in our logistic regression model. Furthermore, only five of 47 TEs in the IBD group occurred postoperatively, five TEs during oral contraceptive use, and none in relation to pregnancies. Thus these risk factors may have only marginally contributed to thromboembolic events in our IBD patients. Finally, we could not elaborate on an association between occurrence of TE and intake of IBD specific medications.

This assumption consequently gives rise to the question of which specific IBD factors might promote the development of TE. It is noteworthy that in the present study, $60 \%$ of TEs in IBD occurred during disease activity or in the presence of complications such as stricture, fistulisation, or abscess. In this respect, the role of endotoxins is highly interesting as endotoxins, with interaction of interleukin 1 and tumour necrosis factor $\alpha(\mathrm{TNF}-\alpha)$, can activate the coagulation cascade $^{27}$ and systemic endotoxinaemia has been detected in active and fistulising CD and can obviously be assumed in the presence of abscesses. ${ }^{28}{ }^{29}$ Furthermore, adding endotoxin to blood samples of patients with IBD induces formation of microclots. ${ }^{30}$ Interestingly, such clot formation cannot be induced in healthy subjects, which suggests that the procoagulatory effect of endotoxins might be enhanced by an IBD specific factor. In fact, it has been suggested that in IBD, activation of the coagulation cascade is more pronounced in active disease. ${ }^{31}$ Additionally, examination of the fibrinolytic system, ${ }^{32}$ platelet count, platelet function, and platelet factors, ${ }^{33}{ }^{34}$ and rheological parameters ${ }^{35}$ have revealed significant abnormalities, which indicate that prothrombotic factors in IBD are more pronounced in active disease. The role of disease activity in promoting TE is further underscored by the report that administration of anti-TNF- $\alpha$ antibodies (infliximab) not only induces clinical remission but also leads to a decrease in activity markers of coagulation. ${ }^{36}$

However, there still remain some thromboembolic events in obviously quiescent IBD, which gives rise to the question of whether IBDs are associated with thrombophilic disorders. Several trials have been performed on the prevalence of resistance to activated protein $\mathrm{C}$ and factor $\mathrm{V}$ Leiden, respectively, factor II polymorphism (20210 G to A), and deficiencies of protein $C$, protein $S$, and antithrombin among patients with IBD. ${ }^{137-41}$ None of these studies was able to prove a specific role for one of these disorders in IBD. In contrast, IBD patients were shown to have higher levels of homocystein more frequently, which was attributed to folate deficiencies secondary to malabsorbtion. ${ }^{42}{ }^{43}$ Conflicting data have been published on the prevalence of antiphospholipid antibodies in IBD, the majority suggesting an increased frequency in IBD. ${ }^{37} 38445$ Finally, CD but not UC patients were shown to have higher levels of liporotein (a) more often compared with controls. ${ }^{46}$ It is known that lipoprotein (a) acts as an acute phase reactant ${ }^{47}$ and it was not reported if lipoprotein (a) was principally increased in these patients or only associated with flares. In summary, many studies have been performed evaluating the role of almost all of the known prothrombotic states in IBD, with none definitively proving any causal relation to the development of TE in IBD.

In conclusion, patients with IBD are at increased risk of developing TE, which seems to be a specific feature of IBD and was not observed in rheumatoid arthritis and coeliac disease. Although there are no data proving a causal relation of any procoagulative state in the promotion of TE in IBD, it is noteworthy that many thromboembolic events occurred during active disease or in the presence of complications such as stricture, fistulisation, or abscess.

\section{ACKNOWLEDGEMENTS}

The study was supported financially by the Anniversary Fund of the Oesterreichische Nationalbank (National Bank Austria) for the additional personnel expenditure necessary for this study (No 7812). This Fund supports scientific research mainly in the fields of economic science and medical science. The Oesterreichische Nationalbank had no influence on the design of the study, collection, analysis, or interpretation of the data, and did not benefit in terms of support or results of this study.

\section{Authors' affiliations}

W Miehsler, W Reinisch, H Vogelsang, G Novacek, Department of Internal Medicine IV, Division of Gastroenterology and Hepatology, University of Vienna, Vienna, Austria

E Valic, W Osterode, Department of Internal Medicine IV, Division of Occupational Medicine, University of Vienna, Vienna, Austria W Tillinger, Department of Internal Medicine 1, Lainz Hospital, Vienna, Austria

T Feichtenschlager, Department of Internal Medicine 4, Rudolfstiffung Hospital, Vienna, Austria

J Grisar, K Machold, Department of Internal Medicine III, Division of Rheumatology, University of Vienna, Vienna, Austria

S Scholz, Department of Medical Statistics, University of Vienna, Vienna, Austria

\section{REFERENCES}

1 Grip O, Svensson P, Lindgren S. Inflammatory bowel disease promotes venous thrombosis earlier in life. Scand J Gastroenterol 2000;35:619-23.

2 Sloan W, Bargen A, Gage R. Life histories of patients with chronic ulcerative colitis: a review of 2000 cases. Gastroenterology 1950;16:25-38.

3 Edwards F, Truelove S. The course and prognosis of ulcerative colitis; Part III Complications. Gut 1964;5:1-15.

4 Talbot R, Heppell J, Dozois R, et al. Vascular complications of inflammatory bowel disease. Mayo Clin Proc 1986;61:140-5.

5 Bernstein C, Blanchard J, Houston D, et al. The incidence of deep venous thrombosis and pulmonary embolism among patients with inflammatory bowel disease: a population based cohort study. Thromb Haemost 2001;85:430-4.

6 Graef V, Baggenstoss A, Saver W, et al. Venous thrombosis occurring in nonspecific ulcerative colitis. Arch Intern Med 1966;117:377-82.

7 Seriolo B, Accardo A, Garnero A, et al. Anticardiolipin antibodies, free protein S levels and thrombosis: a survey in a selected population of rheumatoid arthritis patients. Rheumatology 1999;38:675-8.

8 Young-Hoo K. The incidence of deep vein thrombosis after cementless and cemented knee replacement. J Bone Joint Surg 1990;72:779-83.

9 Pflumio E, Knab M, Muller M, et al. Thrombose splénique et maladie coeliaque: une association fortuite? Presse Med 2000;29:1933-4.

10 Kremer Hovinga J, Baerlocher G, et al. Deep leg vein thrombosis due to acquired thrombophilia-Hyperhomocysteinemia caused by hitherto undiagnosed celiac disease. Ther Umsch 1999;56:519-22.

11 Lennard-Jones J, Shivananda S. Clinical uniformity of inflammatory bowel disease at presentation and during the first year of disease in the north and south of Europe. Eur J Gastroenterol Hepatol 1997:9:353-9.

12 Arnett F, Edworth S, Block D, et al. The American Rheumatism Association 1987 revised criteria for the classification of rheumatoid arthritis. Arthritis Rheum 1988;31:315-24.

13 Walker-Smith J, Guandalini S, Schmitz J, et al. Revised criteria for diagnosis of coeliac disease. Report of working group of European Society of Paediatric Gastroenterology and Nutrition. Arch Dis Child 1990;65:909-11.

14 Lapostolle F, Surget V, Borron S, et al. Severe pulmonary embolism associated with air travel. N Engl J Med 2001;345:779-83.

15 Gotway M, Edinburgh K, Feldstein V, et al. Imaging evaluation of suspected pulmonary embolism. Curr Probl Diagn Radiol 1999;28:129-84.

16 Harvey R, Bradshaw J. A simple index of Crohn's disease activity. Lancet 1980;1:514

17 Truelove S, Witts L. Cortisone in ulcerative colitis. Br Med J 1955;2:1041-8. 
18 Silverstein $M$, Heit J, Moor D, et al. Trends in the incidence of deep venous thrombosis and pulmonary embolism. Arch Intern Med 1998;158:585-93.

19 Heinemann L, Lewis M, Assmann A, et al. Case-control studies on venous thromboembolism: bias due to design? A methodological study on venous thromboembolism and steroid hormone use. Contraception 2002;65:202-14.

20 Stolley P, Strom B. Sample size calculations for clinical pharmacology studies. Clin Pharmacol Ther 1986:39:489-90.

21 Silverberg M, Daly M, Moskovitz D, et al. Diagnostic misclassification reduces the ability to detect linkage in inflammatory bowel disease genetic studies. Gut $2001 ; 49: 773-6$.

22 Farmer M, Petras R, Hunt L, et al. The importance of diagnostic accuracy in colonic inflammatory bowel disease. Am J Gastroenterol 2000;95:3184-8.

23 Farrokhyar F, Swarbrick E, Irvine E. A critical review of epidemiological studies in inflammatory bowel disease. Scand J Gastroenterol 2001;36:2-15.

24 Wallberg-Jonsson S, Johansson H, Öhman ML, et al. Extent of inflammation predicts cardiovascular disease and overall mortality in seropositive rheumatoid arthritis. A retrospective cohort study from disease onset. J Rheumatol 1999;26:2562-71.

25 Heijer M, Koster T, Blom H, et al. Hyperhomocysteinemia as risk factor for deep-vein thrombosis. N Engl J Med 1996:334:759-62.

26 Hansson PO, Eriksson H, Welin L, et al. Smoking and abdominal obesity. Risk factors for venous thromboembolism among middle-aged men: "The study of men born in 1913". Arch Intern Med 1999;159:1886-90.

27 Cybulsky M, Chan W, Movat H. Acute inflammation and microthrombosis induced by endotoxin, interleukin- 1 and tumor necrosis factor and their implication in gram-negative infection. Lab Invest 1988;58:365-78.

28 Gardiner K, Halliday M, Barclay G, et al. Significance of systemic endotoxinaemia in inflammatory bowel disease. Gut 36:897-901

29 Wellmann W, Fink P, Benner F, et al. Endotoxinaemia in active Crohn's disease. Treatment with whole gut irrigation and 5-aminosalicylic acid. Gut 1986;27:814-20

30 Juhlin L, Krause U, Shelley W. Endotoxin-induced microclots in ulcerative colitis and Crohn's disease. Scand J Gastroenterol 1980;15:311-14.

31 Souto J, Martinez E, Roca M et al. Prothrombotic state and signs of endothelial lesion in plasma of patients with inflammatory bowel disease. Dig Dis Sci 1995;40:1883-9.

32 De Jong $E$, Porte R, Knot E, et al. Disturbed fibrinolysis in patients with inflammatory bowel disease. A study in blood plasma, colon mucosa and faeces. Gut 1989;30:188-94.
33 Webberley M, Hart M, Melikian V. Thromboembolism in inflammatory bowel disease: role of platelets. Gut 1993;34:247-51.

34 Collins C, Cahill M, Newland A, et al. Platelets circulate in an activated state in inflammatory bowel disease. Gastroenterology 1994;106:840-5.

35 Novacek G, Vogelsang H, Genser D, et al. Changes in blood rheology caused by Crohn's disease. Eur J Gastroenterol Hepatol 1996;8:1089-93.

36 Hommes D, van Dullemen $H$, Levi $M$, et al. Beneficial effect of treatment with a monoclonal anti-tumor necrosis factor- $\alpha$ antibody on markers of coagulation and fibrinolysis in patients with active Crohn's disease. Haemostasis 1997:27:269-77.

37 Novacek G, Miehsler W, Kapiotis S, et al. Thromboembolism and resistance to activated protein $C$ in patients with inflammatory bowel disease. Am J Gastroenterol 1999;94:685-90.

38 Jackson L, O'Gorman $\mathrm{P}, \mathrm{O}^{\prime}$ Connell J, et al. Thrombosis in inflamatory bowe disease: clinical setting, procoagulant profile and factor $\vee$ Leiden. Q J Med 1997;90:183-8.

39 Guédon C, Le Cam-Duchez V, Lalaude O, et al. Prothrombotic inherited abnormalities other than factor $\mathrm{V}$ Leiden do not play a role in venous thrombosis in inflammatory bowel disease. Am J Gastroenterol 2001;96:1448-54.

40 Vecchi M, Sacchi E, Saibeni S, et al. Inflammatory bowel diseases are not associated with major heriditary conditions predesposing to thrombosis. Dig Dis Sci 2000:45:1465-9.

41 Koutroubakis I, Sfiridaki A, Mouzas I, et al. Resistance to activated protein C and low levels of free protein $S$ in Greek patients with inflammatory bowel disease. Am J Gastroenterol 2000;95:190-4.

42 Cattaneo $M$, Vecchi $M$, Zighetti $M$, et al. High prevalence of hyperhomocysteinemia in patients with inflammatory bowel disease: a pathogenic link with thromboembolic complications? Thromb Haemost 1998;80:542-5.

43 Oldenburg B, Fijnheer R, Griend R, et al. Homocystein in inflammatory bowel disease: a risk factor for thromboembolic complications. Am J Gastroenterol 2000;95:2825-30.

44 Aichbichler B, Petritsch W, Reicht G, et al. Anti-cardiolipin antibodies in patients with inflammatory bowel disease. Dig Dis Sci 1999;44:852-6.

45 Koutroubakis I, Petinaki E, Anagnostopoulou E. Anticardiolipin and anti beta2-glycoprotein I antibodies in patients with inflammatory bowel disease. Dig Dis Sci 1998;43:2507-12.

46 Hudson $M$, Chitolie A, Hutton R, et al. Thrombotic vascular risk factors in inflammatory bowel disease. Gut 1996;38:733-7.

47 Maeda S, Abe A, Seishima M, et al. Transient changes of serum lipoprotein (a) as an acute phase protein. Atherosclerosis 1989;78:145-50. 